\title{
Influence of dialysis duration and parathyroid hormone on the clinical and radiographic oral conditions of pre-transplant patients with chronic kidney disease
}

\author{
Stênio Medeiros Queiroz', Adriana Gomes Amorim², Ana Luiza Dias Leite de Andrade², \\ Manuel Antonio Gordón-Núñez ${ }^{2}$, Roseana de Almeida Freitas'², Hébel Cavalcanti Galvão ${ }^{1}$
}

${ }^{1}$ Center of Health Sciences, Rio Grande do Norte Federal University, Natal, RN, Brazil

${ }^{2}$ Area of Oral Pathology, Department of Dentistry, Rio Grande do Norte Federal University, Natal, RN, Brazil

\begin{abstract}
Aim: To evaluate the oral conditions of patients with chronic kidney disease undergoing dialysis and to determine the influence of dialysis duration and bone metabolism on the prevalence and severity of the alterations found. Methods: The simplified oral hygiene index (OHI-S), prevalence of dental caries (decayed, missing filled teeth index, DMFT), and the periodontal screening and recording (PSR) index were evaluated in 154 patients. Parathyroid hormone (PTH), calcium, phosphorus and urea measurements, as well as panoramic radiographs, were obtained from all patients. To evaluate the effect of duration of dialysis treatment on oral health, the patients were divided into two groups: (1) $<5$ years and (2) $\geq 5$ years. Regarding blood levels of PTH, patients were divided into three groups: (1) 0-149 pg/mL, (2) $150-584 \mathrm{pg} / \mathrm{mL}$, and (3) $>585 \mathrm{pg} / \mathrm{mL}$. The $\mathrm{OHI}-\mathrm{S}$ identified the accumulation of biofilm and calculus around the teeth. Results: Gingival inflammation was found in $100 \%$ of dentate patients, with 2 being the predominant PSR score (72.3\%). The DMFT index was high (17.52). Dental calculus was the most common radiographic finding $(70.8 \%)$. No significant correlation was observed between dialysis duration, biochemical alterations, and oral health. Conclusions: Most patients undergoing dialysis presented precarious oral hygiene, periodontal inflammation, and bone alterations. However, these manifestations were not influenced by the duration of dialysis or bone metabolism.
\end{abstract}

Keywords: chronic kidney disease, parathyroid hormone, oral health, hemodialysis.

Received for publication: March 11, 2013 Accepted: June 25, 2013

Correspondence to: Hébel Cavalcanti Galvão Avenida General Gustavo Cordeiro de Farias, s/n, CEP: 59010-180 - Petrópolis,

Natal, RN, Brasil

Phone/Fax: +55 $8433429776 / 32154108$

E-mail: hebel.galvao@yahoo.com.br

\section{Introduction}

Chronic kidney disease (CKD) is a global public health problem. The disease is characterized by a slow, progressive and irreversible decline in the number of functional nephrons, which results in a decrease of glomerular filtration rate and in the accumulation of various, often toxic, substances that are normally excreted by the kidneys, causing uremic syndrome $\mathrm{e}^{1-5}$, as well as disturbances in normal homeostatic mechanisms that control the water-electrolyte balance in the 
organism $^{1}$. Diabetes mellitus, hypertension, glomerulonephritis, and renal cystic disease are the most common causes of kidney failure, whose incidence and prevalence have increased over the last few years ${ }^{2}$. CKD is defined when kidney function decreases by 5 to $10 \%$ of its original capacity. Treatment includes hemodialysis (HD) or continuous ambulatory peritoneal dialysis (CAPD) as replacement of renal filtration, and a kidney transplant as definitive therapy ${ }^{6}$.

Patients with CKD show a broad spectrum of oral manifestations that affect the soft or hard tissues ${ }^{7}$, including xerostomia, uremic breath, uremic stomatitis, radiographic changes of the jaw bones, accumulation of calculus on the teeth, periodontitis, and other less frequent abnormalities ${ }^{2-}$ 3,8 . Some of these conditions are more severe in patients undergoing dialysis than in healthy individuals ${ }^{9-12}$.

The duration of dialysis can influence the prevalence of oral abnormalities observed may be related to worsening of oral health ${ }^{11,13}$. With respect to bone alterations commonly observed in these individuals, bone metabolism disorders caused by altered blood levels of calcium $(\mathrm{Ca})$ and phosphorus (P) in response to lack of activation of vitamin $\mathrm{D}$ by the kidneys promote the development of secondary hyperparathyroidism, contributing to alveolar bone loss by changing the normal skeletal remodeling, in addition to favoring the development of intra-osseous lesions such as brown tumor ${ }^{14}$.

These oral conditions can become a source of infection since patients with CKD are highly susceptible due to the immunodeficiency caused by uremia ${ }^{3}$. After transplantation, the persistence of oral infections may be a cause of morbidity in patients on immunosuppressive therapy ${ }^{10}$. It is therefore important that dentists are aware of the main conditions affecting the oral cavity of these patients in order to prevent or treat them before transplantation ${ }^{4}$. The objective of the present cross-sectional cohort study was to evaluate cross through clinical, biochemical and radiographic oral conditions in a Brazilian population with CKD that was preparing for a kidney transplant, investigating the influence of dialysis duration and status on bone metabolism the prevalence and severity of the changes found.

\section{Material and methods}

The sample consisted of 154 patients of both genders with CKD undergoing HD or CAPD for at least 3 months. The patients were recruited from the Central Preparation Center for Kidney Transplantation, Onofre Lopes University Hospital (Rio Grande do Norte, Brazil), were older than 18 years, and had not received dental treatment during the previous 6 months. The study was conducted in accordance with the Declaration of Helsinki's guidelines and was approved by the Ethics Committee of Federal University of Rio Grande do Norte (UFRN) (Protocol 082/09). All patients gave written informed consent to participate in the study.

A clinical form containing the following data was completed for each patient: demographic data; data about the cause of CKD; type, duration and place of dialysis; medications used; results of routine biochemical tests; frequency of toothbrushing; use of dental floss; presence of bitter taste in the mouth; xerostomia; use of dentures, and date of the last visit to the dentist.

The patients underwent intraoral clinical examination at the dental office of the Family and Community Health Unit of Onofre Lopes University Hospital, UFRN, using a dental mirror, exploratory probe, basic fuchsine (Eviplac ${ }^{\circledR}$ ) for biofilm detection, and a periodontal probe (OMS-621). This examination was performed to evaluate the presence of alterations in oral soft tissues (lip, palate, tongue, floor of the mouth, cheek mucosa) such as candidiasis and petechiae. Next, the teeth were examined regarding the presence of attrition, erosion, abrasion, mobility, stains and hypoplasia.

The prevalence of caries was analyzed using the decayed, missing, filled teeth (DMFT) index recommended by the World Health Organization. Oral hygiene was evaluated using the simplified oral hygiene index (OHI-S) proposed by Greene and Vermilion ${ }^{15}$ (1964), which combines two components: biofilm index and calculus index. Six sites of selected teeth were examined after application of a biofilm stain: buccal surface of teeth $16,26,11$ and 31 , and lingual surface of teeth 36 and 46. A score of 0 to 3 was attributed to each surface according to the amount of biofilm or calculus detected. For the biofilm index, scores were attributed as follows: 0 (no staining), 1 (biofilm covering no more than $1 / 3$ of the tooth surface), 2 (biofilm covering more than $1 / 3$ and less than $2 / 3$ of the tooth surface), and 3 (biofilm covering more than 2/ 3 of the tooth surface). The following scores were attributed for determination of the calculus index: 0 (no calculus), 1 (supragingival calculus covering no more than $1 / 3$ of the tooth surface), 2 (supragingival calculus covering more than $1 / 3$ and less than $2 / 3$ of the tooth surface), and 3 (calculus covering more than $2 / 3$ of the tooth surface, or a band of subgingival calculus in the cervical region of the tooth). The OHI-S is calculated as the sum of scores of the two components.

The periodontal screening and recording (PSR) system was used to analyze the need for periodontal treatment. For this system, the mouth is divided into sextants and the tip of the periodontal probe is inserted into the gingival sulcus of the teeth present. All tooth surfaces are examined and the highest score is recorded. The following scores are attributed:

0 : Colored part of the probe completely visible. Absence of calculus and defective restorations. Healthy periodontal tissues.

1: Gingivitis. Colored part of the probe completely visible but bleeding on probing. Absence of calculus and defective restorations.

2: Gingivitis or mild periodontitis. Colored part of the probe completely visible but bleeding on probing. Presence of supra- or subgingival calculus.

3: Moderate periodontitis. Colored part of the probe partially visible, with a pocket depth of 4 to $5 \mathrm{~mm}$.

4: Advanced periodontitis. Colored part of the probe disappearing completely inside the gingival pocket.

Clinical evaluation was performed by a single calibrated examiner. Kappa statistics was used to evaluate the level of intraexaminer agreement for OHI-S, DMFT, and PSR. 
Calibration was performed on 10 patients at an interval of one hour between analyses. All kappa values were close to 0.70 , indicating almost perfect agreement. Panoramic radiographs were taken from all patients to identify sources of infection, the presence of calculus, and bone alterations. All participants received detailed instructions and were encouraged to practice oral hygiene. Patients who required dental treatment were referred to the Department of Dentistry of UFRN where they underwent the adequate procedures.

Routine biochemical parameters obtained at the dialysis centers, including parathyroid hormone (PTH), Ca, P, and pre and post-dialysis urea, were used to evaluate the correlation between bone and mineral metabolism, which is commonly altered in this group of patients, and the clinical and radiographic alterations observed in this study. Since PTH concentration indicates the presence of bone disease, the patients undergoing dialysis were divided into three groups according to PTH levels: group 1: 0 to $149 \mathrm{pg} / \mathrm{mL}$ (low turnover bone disease); group 2: 150 to $584 \mathrm{pg} / \mathrm{mL}$ (absence of bone disease); group 3: > $585 \mathrm{pg} / \mathrm{mL}$ (high turnover bone disease). The $\mathrm{Ca} \times \mathrm{P}$ product was calculated for each patient and the sample was divided into two groups according to the risk of extraskeletal metastatic calcifications: group 1 ( $\mathrm{Ca} \times \mathrm{P}<55 \mathrm{mg} / \mathrm{dL}$ ) and group 2 ( $\mathrm{Ca} \times \mathrm{P} \geq 55 \mathrm{mg}$ / $\mathrm{dL}$ ). In addition, the urea reduction ratio (URR) was obtained for each participant to evaluate the efficiency of dialysis. A URR $>65 \%$ was considered to indicate adequate dialysis.

For determination of the effect of dialysis duration on DMFT, OHI-S, PSR, radiographic bone alterations, dental calculus, $\mathrm{Ca} \times \mathrm{P}$ product, and $\mathrm{PTH}$, the patients undergoing dialysis were divided into two groups: group 1 consisting of patients undergoing dialysis for $<5$ years, and group 2 consisting of patients undergoing dialysis for $\geq 5$ years.

The data were entered into Microsoft Excel spreadsheets and analyzed using the SPSS 17.0 software (SPSS Inc., Chicago, IL, USA). Variables are expressed as absolute (n) and relative $(\%)$ frequencies and as means. The KolmogorovSmirnov test was used to determine whether numerical variables showed a normal distribution. Differences between normally distributed quantitative variables were evaluated by the Student $t$-test. Associations between qualitative variables were tested by the chi-squared test or Fisher's exact test. Mean and median biochemical parameters were compared between groups using the nonparametric Kruskal-Wallis test for PTH and the Mann-Whitney test for URR and $\mathrm{Ca}$ x P product. A level of significance of 5\% (0.05) was adopted.

\section{Results}

The mean age was 41.8 years. Patients aged 36 to 45 years were the predominant age group, corresponding to $22.7 \%$. Most patients were males $(55.8 \%)$ and $46.1 \%$ were Caucasian. Hypertension $(43.50 \%)$ was the most common causes of CKD, followed by unspecified chronic glomerulonephritis $(9.74 \%)$. Hemodialysis was the most frequent type of dialysis $(92.2 \%)$ and $94(61 \%)$ patients with CKD had started treatment at least 5 years earlier. Sixteen (10.4\%) of the 154 patients were completely edentulous and 59 (38.3\%) used some type of denture. With respect to oral hygiene habits, $53.9 \%$ of the patients brushed their teeth three times per day; however, only $24.7 \%$ used dental floss regularly. At the time of the present study, $44.8 \%$ of the participants reported to have visited the dentist in the previous year. With respect to the use of vitamins and medications for the control of plasma calcium and potassium levels, the patients also reported the use of other drugs, including antihypertensives (55.8\%), hypoglycemics (5.8\%), antiulcer drugs $(14.3 \%)$, and anxiolytics $(5.2 \%)$.

The most frequent oral clinical finding was coated tongue (31.2\%), which is generally associated with inadequate hygiene. Other changes observed in soft tissues were petechiae $(8.5 \%)$, candidiasis $(2.6 \%)$, nicotine stomatitis $(1.3 \%)$, hemangioma $(1.3 \%)$, fibroma $(1.3 \%)$, fibrous hyperplasia $(1.3 \%)$, and actinic cheilitis $(0.6 \%)$. Xerostomia was reported by $42.9 \%$ of the patients and altered taste by $37 \%$. Attrition was the most common dental problem $(48.5 \%)$, followed by abrasion $(23.2 \%)$ and tooth mobility (15.2\%). Bruxism was present in 21 (13.6\%) patients.

The most frequent radiographic alteration was dental calculus, observed in $109(70.8 \%)$ patients. Other findings were dental caries $(62.3 \%)$, horizontal bone loss $(62.3 \%)$, vertical bone loss $(39.6 \%)$, residual roots $(29.9 \%)$, lost teeth (85.1\%), and impacted teeth (19.5\%). Signs of pulp necrosis, such as diffuse bone rarefaction were observed in 28 (18.2\%) patients and circumscribed bone rarefaction in $9(5.8 \%)$ (Table 1). One patient had a brown tumor. Vertical and horizontal bone loss was significantly correlated with age $(p=0.004$

Table 1. Soft and hard tissue alterations in the oral cavity and radiographic findings in patients undergoing dialysis.

\begin{tabular}{|c|c|c|}
\hline Variables & & $\mathrm{n}(\%)$ \\
\hline Location of oral soft & Jugal mucosa & $16(10.4)$ \\
\hline \multirow[t]{5}{*}{ tissue alterations } & Hard palate & $6(3.9)$ \\
\hline & Lip & $6(3.9)$ \\
\hline & Petechiae & $13(8.5)$ \\
\hline & Candidiasis & $4(2.6)$ \\
\hline & Nicotine stomatitis & $2(1.3)$ \\
\hline \multirow[t]{6}{*}{ Oral soft tissue alterations } & Actinic cheilitis & $1(0.6)$ \\
\hline & Hemangioma & $2(1.3)$ \\
\hline & Fibroma & $2(1.3)$ \\
\hline & Fibrous hyperplasia & $2(1.3)$ \\
\hline & Attrition & $48(48.5)$ \\
\hline & Abrasion & $23(23.2)$ \\
\hline \multirow[t]{7}{*}{ Tooth alterations } & Mobility & $15(15.2)$ \\
\hline & Erosion & $6(6.1)$ \\
\hline & Stains & $5(5.1)$ \\
\hline & Hypoplasia & $2(1.3)$ \\
\hline & Calculus & $109(70.8)$ \\
\hline & Tooth decay & $96(62.3)$ \\
\hline & Horizontal bone loss & $96(62.3)$ \\
\hline \multirow[t]{5}{*}{ Radiographic alterations } & Vertical bone loss & $61(39.6)$ \\
\hline & Residual roots & $46(29.9)$ \\
\hline & Impacted teeth & $30(19.5)$ \\
\hline & Diffuse bone rarefaction & $28(18.2)$ \\
\hline & Circumscribed bone rarefaction & $9(5.8)$ \\
\hline
\end{tabular}


and $p<0.001$, respectively).

Analysis of oral hygiene conditions in dentate patients using the OHI-S showed deficient hygiene in $36.8 \%$ of these patients. Evaluation of the need for periodontal treatment revealed a PSR score of 2 in $72.3 \%$ of the patients. These results indicate the presence of supra- or subgingival calculus and bleeding on probing. PSR was not performed in one patient and the OHI-S was not obtained in five because they did not attend the scheduled exam. The chi-squared and Fisher's exact tests revealed no significant association of dialysis duration, OHI-S, PSR, PTH or Ca x P product with radiographic bone alterations (vertical and horizontal bone resorption, dental calculus, diffuse and circumscribed bone rarefaction, bone sclerosis, and bone repair) (Table 2). The mean DMFT was $17.52(\mathrm{D}=2.29, \mathrm{M}=11.87$, and $\mathrm{F}=$ 3.36). Analysis of DMFT according to the duration of dialysis showed a mean $( \pm$ standard deviation) index of $16.8( \pm$ $9.8)$ in the group $<5$ years of dialysis and of $18.5( \pm 8.1)$ in the group $\geq 5$ years of dialysis, but the difference was not significant $(\mathrm{p}=0.268$, Student t-test).

There was no significant association between the three PTH ranges and radiographic bone alterations (calculus, circumscribed and diffuse bone rarefaction, sclerosis, bone repair, horizontal and vertical bone loss, and periapical lesion). In addition, no association was observed between this biochemical parameter and the calculus component of the OHI-S (Table 3). The latter was also not significantly associated with the $\mathrm{Ca} \times \mathrm{P}$ product $(\mathrm{p}=0.404)$, or with any of the bone alterations apparent on panoramic radiographs. The frequency of calculus demonstrated by radiography was higher in patients with a URR $<65 \%$, but this difference was not statistically significant $(p=0.579)$. None of the biochemical parameters studied was correlated with periodontal disease based on PSR score.

\section{Discussion}

The therapeutic modalities for patients with CKD such as dialysis increase the life expectancy of this population. However, patients undergoing dialysis are more susceptible to infections due to their state of immunodeficiency. These patients, particularly transplant candidates, should therefore be submitted to careful oral examination to eliminate any source of infection. In the present study, 154 pre-transplant patients with CKD undergoing dialysis were examined to identify oral conditions associated with the disease. In addition, the influence of dialysis duration and bone and mineral metabolism on the oral manifestations of these patients was evaluated.

Descriptive analysis showed that coated tongue was the most common oral clinical finding in soft tissues, a condition generally associated with inadequate hygiene. In addition, there was a high prevalence of dry mouth, a finding that might be related to the restricted intake of fluids by patients undergoing dialysis or to the use of medications. Similar results have been reported by Dirschnabel et al. ${ }^{7}$. Petechiae were observed in $16.4 \%$ of the sample and might be related
Table 2. Comparison of OHI-S, PSR, DMFT, biochemical parameters ( $\mathrm{PTH}, \mathrm{Ca} \times \mathrm{P}$ product, pre-dialysis urea), and radiographic findings (diffuse and circumscribed bone rarefaction, sclerosis, bone repair, dental calculus, vertical and horizontal bone loss) between patients undergoing dialysis for $<5$ years and $\geq 5$ years.

\begin{tabular}{|c|c|c|c|}
\hline \multirow{3}{*}{ OHI-S } & \multicolumn{2}{|c|}{ Time of dialysis } & \multirow{2}{*}{$p$ value } \\
\hline & \multirow[t]{2}{*}{$<5$ years } & \multirow[t]{2}{*}{$\geq 5$ years } & \\
\hline & & & \\
\hline Satisfactory & $10(7.5 \%)$ & $7(5.3 \%)$ & $0.910^{1}$ \\
\hline Regular & $24(18 \%)$ & $15(11.3 \%)$ & \\
\hline Deficient & $30(22.6 \%)$ & $19(14.3 \%)$ & \\
\hline Very poor & $15(11.3 \%)$ & $13(9.8 \%)$ & \\
\hline \multicolumn{4}{|l|}{ PSR } \\
\hline Grade 1 & $5(3.6 \%)$ & $2(1.5 \%)$ & $0.780^{1}$ \\
\hline Grade 2 & $61(44.5 \%)$ & $39(28.5 \%)$ & \\
\hline Grade 3 & $13(9.5 \%)$ & $12(8.8 \%)$ & \\
\hline Grade 4 & $3(2.2 \%)$ & $2(1.5 \%)$ & \\
\hline \multicolumn{4}{|l|}{ DMFT } \\
\hline Total & $16.8 \pm 9.8$ & $18.5 \pm 8.1$ & $0.268^{2}$ \\
\hline \multicolumn{4}{|l|}{ PTH } \\
\hline 0 a 149 & $27(17.8 \%)$ & $20(13.2 \%)$ & $0.733^{1}$ \\
\hline 150 a 584 & $42(27.6 \%)$ & $23(15.1 \%)$ & \\
\hline $585+$ & $24(15.8 \%)$ & $16(10.5 \%)$ & \\
\hline \multicolumn{4}{|l|}{$\mathrm{Ca} \times \mathrm{P}$} \\
\hline$<55$ & $54(35.1 \%)$ & $36(23.4 \%)$ & $0.754^{1}$ \\
\hline$\geq 55$ & $40(26 \%)$ & $24(15.6 \%)$ & \\
\hline \multicolumn{4}{|l|}{ URR } \\
\hline$<65 \%$ & $21(13.8 \%)$ & $11(7.2 \%)$ & $0,620^{1}$ \\
\hline$\geq 65 \%$ & $73(48 \%)$ & $47(30.9 \%)$ & \\
\hline \multicolumn{4}{|c|}{ Pre-dialysis urea } \\
\hline 15 a 100 & $3(2 \%)$ & $0(0 \%)$ & $0.229^{1}$ \\
\hline 101 a 200 & $82(53.9 \%)$ & $49(32.2 \%)$ & \\
\hline$>200$ & $9(5.9 \%)$ & $9(5.9 \%)$ & \\
\hline \multicolumn{4}{|c|}{ Diffuse bone rarefaction } \\
\hline Yes & $15(9.7 \%)$ & $13(8.4 \%)$ & $0.370^{1}$ \\
\hline No & $79(51.3 \%)$ & $47(30.5 \%)$ & \\
\hline \multicolumn{4}{|c|}{ Circumscribed bone rarefaction } \\
\hline Yes & $6(3.9 \%)$ & $3(1.9 \%)$ & $0.508^{3}$ \\
\hline No & $88(57.1 \%)$ & $57(37.0 \%)$ & \\
\hline \multicolumn{4}{|l|}{ Bone sclerosis } \\
\hline Yes & $1(0.6 \%)$ & $1(0.6 \%)$ & $0.629^{3}$ \\
\hline No & $93(60.4 \%)$ & $59(38.3 \%)$ & \\
\hline \multicolumn{4}{|l|}{ Bone repair } \\
\hline Yes & $11(7.1 \%)$ & $12(7.8 \%)$ & $0.159^{1}$ \\
\hline No & $83(53.9 \%)$ & $48(31.2 \%)$ & \\
\hline \multicolumn{4}{|c|}{ Vertical bone loss } \\
\hline Yes & $34(22.1 \%)$ & $27(17.5 \%)$ & $0.275^{1}$ \\
\hline No & $60(39.0 \%)$ & $33(21.4 \%)$ & \\
\hline \multicolumn{4}{|c|}{ Horizontal bone loss } \\
\hline Yes & $55(35.7 \%)$ & $41(26.6 \%)$ & $0.220^{1}$ \\
\hline No & $39(25.3 \%)$ & $19(12.3 \%)$ & \\
\hline \multicolumn{4}{|l|}{ Dental calculus } \\
\hline Yes & $68(44.2 \%)$ & $41(26.6 \%)$ & $0.594^{1}$ \\
\hline No & $26(16.9 \%)$ & $19(12.3 \%)$ & \\
\hline
\end{tabular}

1. $p$ value of chi-square test

2. $p$ value of the Student's $t$ test.

3. $p$ value of Fischer's exact test. 
Table 3. Absolute and relative frequencies of the main radiographic findings, PSR score and calculus component of the OHI-S according to parathyroid hormone levels.

\begin{tabular}{|c|c|c|c|c|}
\hline & \multicolumn{3}{|c|}{ PTH $^{1}$} & \multirow[t]{2}{*}{$p$} \\
\hline & 0 a 149 & 150 a 584 & $\geq 585$ & \\
\hline \multicolumn{5}{|c|}{ Diffuse bone rarefaction } \\
\hline Yes & $13(8.6 \%)$ & $8(5.3 \%)$ & $6(3.9 \%)$ & \multirow[t]{2}{*}{$0.096^{2}$} \\
\hline No & $34(22.4 \%)$ & $57(37.5 \%)$ & $34(22.4 \%)$ & \\
\hline \multicolumn{5}{|c|}{ Circumscribed bone rarefaction } \\
\hline Yes & $3(2.0 \%)$ & $5(3.3 \%)$ & $1(0.7 \%)$ & \multirow[t]{2}{*}{$0.542^{3}$} \\
\hline No & $44(28.9 \%)$ & $60(39.5 \%)$ & $39(25.7 \%)$ & \\
\hline \multicolumn{5}{|l|}{ Bone sclerosis } \\
\hline Yes & $1(0.7 \%)$ & $0(0 \%)$ & $1(0.7 \%)$ & \multirow[t]{2}{*}{$0.464^{3}$} \\
\hline No & $46(30.3 \%)$ & $65(42.8 \%)$ & $39(25.7 \%)$ & \\
\hline \multicolumn{5}{|l|}{ Bone repair } \\
\hline Yes & $6(3.9 \%)$ & $12(7.9 \%)$ & $4(2.6 \%)$ & \multirow[t]{2}{*}{$0.451^{3}$} \\
\hline No & $41(27.0 \%)$ & $53(34.9 \%)$ & $36(23.7 \%)$ & \\
\hline \multicolumn{5}{|l|}{ Vertical bone loss } \\
\hline Yes & $19(12.5 \%)$ & $27(17.8 \%)$ & $14(9.2 \%)$ & \multirow[t]{2}{*}{$0.791^{2}$} \\
\hline No & $28(18.4 \%)$ & $38(25.0 \%)$ & $26(17.1 \%)$ & \\
\hline \multicolumn{5}{|c|}{ Horizontal bone loss } \\
\hline Yes & $32(21.1 \%)$ & $35(23.0 \%)$ & $27(17.8 \%)$ & \multirow[t]{2}{*}{$0.214^{2}$} \\
\hline No & $15(9.9 \%)$ & $30(19.7 \%)$ & $13(8.6 \%)$ & \\
\hline \multicolumn{5}{|l|}{ Calculus } \\
\hline Yes & $36(23.7 \%)$ & $41(27.0 \%)$ & $30(19.7 \%)$ & \multirow[t]{2}{*}{$0.229^{2}$} \\
\hline No & $11(7.2 \%)$ & $24(15.8 \%)$ & $10(6.6 \%)$ & \\
\hline \multicolumn{5}{|l|}{ PSR } \\
\hline Grade 1 & $2(1.5 \%)$ & $4(3.0 \%)$ & $1(0.7 \%)$ & \multirow[t]{4}{*}{$0.646^{2}$} \\
\hline Grade 2 & $31(21.0 \%)$ & $44(32.6 \%)$ & $24(17.8 \%)$ & \\
\hline Grade 3 & 7 (5.2\%) & 8 (5.9\%) & $10(7.4 \%)$ & \\
\hline Grade 4 & $2(1.5 \%)$ & $1(0.7 \%)$ & $1(0.7 \%)$ & \\
\hline OHI-S calculus & $0.82 \pm 0.6$ & $0.83 \pm 0.65$ & $0.82 \pm 0.6$ & $0.966^{4}$ \\
\hline
\end{tabular}

Group 1: 0 to $149 \mathrm{pg} / \mathrm{mL}$ (low turnover bone disease); group 2: 150 to $584 \mathrm{pg} / \mathrm{mL}$ (absence of bone disease); group 3: > $585 \mathrm{pg} /$ $\mathrm{mL}$ (high turnover bone disease).

1. $p$ value of chi-square.

2. $p$ value of Fischer's exact test.

3. $p$ value of the Kruskal-Wallis.

to the use of heparin during the HD sessions. Halitosis and altered taste can be the result of xerostomia and the presence of oral microorganisms that metabolize urea (found at high levels in the saliva of these patients) and produce ammonia. About one-third of patients undergoing dialysis complain of a bad taste in their mouth ${ }^{2,6}$ in agreement with the present study in which $37 \%$ of the sample reported this symptom. Attrition was the most common dental problem. According to Klassen and $\mathrm{Krasco}^{16}$ this condition is secondary to aging or xerostomia.

Most patients studied here presented poor oral hygiene conditions. Souza ${ }^{3}$ and Gürkan et al. ${ }^{4}$ suggested that some oral problems may occur due to the negligence of preventive health measures since the patients are more concerned with the treatment of CKD. However, since the present patients reported a good frequency of daily tooth brushing, the precarious oral conditions might have been due to the lack of knowledge about adequate brushing techniques. In addition, most patients did not use dental floss, a fact favoring biofilm accumulation on the interproximal surfaces of teeth. The OHI-S also indicated the abundant presence of calculus in these patients, a fact contributing to the high scores observed. A high concentration of salivary urea can influence the formation of calculus ${ }^{3,6}$. Other factors that favor the development of calculus in these patients are the intake of calcium supplements, hyperparathyroidism, and inadequate hygiene ${ }^{17}$. Although almost half the sample $(44.8 \%)$ had seen a dentist during the year prior to the study, this fact did not improve oral hygiene since this care focused on isolated treatment and not on prevention. These results highlight the need to invest in oral health promotion programs for this population.

A high DMFT index (17.52) was found in the sample studied. Similar results have been reported by other investigators ${ }^{6,17}$ who observed no significant differences in DMFT indices between patients with CKD and control groups. The high DMFT index observed in the present study was mainly due to the large number of missing teeth (11.87) when compared to decayed (2.29) and filled teeth (3.36). According to Dias et al. ${ }^{18}$ these findings are not directly related to kidney disease, but rather to the socioeconomic conditions of the patients and limited access to preventive/ curative treatment. As a consequence, the patient only sought 
treatment when experiencing pain and tooth extraction was frequently required in these cases.

With respect to periodontal disease, Chamani et al. ${ }^{12}$ observed some degree of gingival inflammation in all patients studied, in agreement with the present investigation in which $100 \%$ of dentate patients had gingivitis, with a predominance of PSR score 2 (72.3\%). Periodontal disease is highly prevalent and severe in patients with kidney failure. Prophylaxis and early dental treatment should be reinforced and may have a positive impact on the general health status of the patient ${ }^{19}$.

The investigation of sources of infection was complemented by panoramic radiography, which permitted the visualization of the teeth and their supporting bone structures. Studies using radiographic analysis for CKD patients undergoing dialysis are scarce in the literature. In the present study, calculus was the most common radiographic alteration, followed by dental caries. Thorman et al. ${ }^{20}$ compared the panoramic radiographs of 93 patients with CKD (pre-dialysis, HD, and CAPD) with a control group and found a higher incidence of periapical infections in the first three groups. These results highlight the importance of radiographic investigation in all pre-transplant patients undergoing dialysis since they may present inflammatory lesions of the teeth that cannot be detected by clinical examination alone.

PTH concentration can be used as a biochemical marker of bone remodeling. An increase of this hormone occurs in response to altered blood levels of $\mathrm{Ca}$ and $\mathrm{P}$ in the organism caused by the loss of renal function. This condition, in turn, leads to secondary hyperparathyroidism, which promotes the development of high turnover bone disease. On the other hand, renal osteodystrophy can manifest as a low turnover bone disease associated with low PTH levels, particularly in diabetic patients ${ }^{21}$. In the present study, no correlation was observed between blood PTH levels and bone alterations observed on panoramic radiographs in any of the three groups studied. Frankenthal et al. ${ }^{14}$ also found no influence of secondary hyperparathyroidism on alveolar bone loss or periodontal indices in 35 patients with CKD undergoing dialysis. In addition, URR or $\mathrm{Ca} \times \mathrm{P}$ product was not associated with calculus accumulation, periodontal disease or bone loss. These data indicate that bone and mineral metabolism, which is frequently altered in patients undergoing dialysis, did not influence the oral alterations observed in this study.

Analysis of the effect of dialysis duration on the oral conditions of patients with CKD showed no significant difference in PSR, OHI-S or DMFT between the two groups studied ( $<5$ years and $\geq 5$ years). These results agree with other investigators who suggested that the duration of dialysis exerts no additional effect on dental or periodontal conditions in patients with $\mathrm{CKD}^{12}$. In contrast, some studies reported a higher accumulation of biofilm and calculus and a high degree of gingival bleeding in long-term patients undergoing dialysis ${ }^{9,11,13}$. Bone and mineral metabolism evaluated based on blood PTH concentrations and $\mathrm{Ca} \times \mathrm{P}$ product was also not influenced by the duration of dialysis. No significant difference in radiographic bone alterations was observed between the two groups $(<5$ years and $\geq 5$ years). We found no studies in the literature investigating the influence of dialysis duration on oral conditions detected by panoramic radiography.

Most patients with CKD undergoing dialysis presented poor oral hygiene, calculus accumulation and gingival inflammation, which represent sources of infection. However, the duration of dialysis was not associated with poor oral conditions. The state of bone or mineral metabolism also did not influence oral health manifestations in the patients studied. Taken together, the results indicate the need for oral health care in these patients as an important part of the kidney transplant preparation protocol.

\section{Acknowledgements}

The authors thank the Foundation for Research Support of the State of Rio Grande do Norte, Brazil, for financial support.

\section{References}

1. Marakoglu I, Gursoy UK, Demirer S, Sezer H. Periodontal status of chronic renal failure patients receiving hemodialysis. Yonsei Med J. 2003; 44: 648-52

2. Proctor R, Kumar N, Stein A, Moles D, Porter S. Oral and dental aspects of chronic renal failure. J Dent Res. 2005; 84: 199-208.

3. Souza CRD, Libério SA, Guerra RNM, Monteiro S, Silveira EJD, Pereira ALA. Evaluation of periodontal condition of kidney patients on dialysis. Rev Assoc Med Bras. 2005; 51: 285-9.

4. Gürkan A, Köse T, Atilla G. Oral Health Status and Oral Hygiene Habits of an Adult Turkish Population on Dialysis. Oral Health Prev Dent. 2008; 6: 37-43.

5. Carvalho AA, Farsura PP, Bastos MG, Vilela EM. Influence of nonsurgical periodontal treatment on hematological and biochemical parameters of patients with chronic renal failure in pre-dialysis. Periodontia. 2011; 21: 27-33.

6. Bots CP, Poorterman JHG, Brand HS, Kalsbeek H, van Amerongen BM, Veerman EC et al. The oral health status of dentate patients with chronic renal failure undergoing dialysis therapy. Oral Dis. 2006; 12: 176-80.

7. Dirschnabel AJ, Martins AS, Dantas SAG, Ribas Mde O, Grégio AM, Alanis LR et al. Clinical oral findings in dialysis and kidney-transplant patients. Quintessence Int. 2011; 42: 127-33.

8. Kho H, Lee S, Sung-Chang Chung S, Kim Y. Oral manifestations and salivary flow rate, $\mathrm{pH}$, and buffer capacity in patients with end-stage renal disease undergoing hemodialysis. Oral Surg Oral Med Oral Pathol Oral Radiol Endod. 1999; 88: 316-9.

9. Bayraktar G, Kurtulus I, Duraduryan A, Cintan S, Kazancioglu R, Yildiz A et al. Dental and periodontal findings in hemodialysis patients. Oral Dis. 2007; 13: 393-7.

10. Bayraktar G, Kurtulus I, Kazancioglu R, Bayramgurler I, Cintan S, Bural Cetal. Evaluation of periodontal parameters in patients undergoing peritoneal dialysis or hemodialysis. Oral Dis. 2008; 14: 185-9.

11. Cengiz MI, Sümer P, Cengiz S, Yavuz U. The effect of the duration of the dialysis in hemodialysis patients on dental and periodontal findings. Oral Dis. 2009; 15: 336-41.

12. Chamani G, Zarei MR, Radvar M, Rashidfarrokhi F, Razazpour F. Oral health status of dialysis patients based on their renal dialysis history in Kerman, Iran. Oral Health Prev Dent. 2009; 7: 269-75.

13. Sekiguchi RT, Pannuti CM, Silva Jr HT, Medina-Pestana JO, Romito GA. Decrease in oral health may be associated with length of time since beginning dialysis. Spec Care Dent. 2012; 32: 6-10. 
14. Frankenthal S, Nakhoul F, Machtei EE, Green J, Ardekian L, Laufer D et al. The effect of secondary hyperparathyroidism and hemodialysis therapy on alveolar bone and periodontium. J Clin Periodontol. 2002; 29: 479-83.

15. Greene JC, Vermillion JR. The simplified oral hygiene index. J Am Dent Assoc. 1964; 68: 7-13.

16. Klassen JT, Krasko BM. The dental health status of dialysis patients. J Can Dent Assoc. 2002; 68: 34-8.

17. Souza CM, Braosi APR, Luczyszyn SM, Casagrande RW, PecoitsFilho R, Riella MC et al. Oral health in Brazilian patients with chronic renal disease. Rev Med Chil. 2008; 136: 741-6.

18. Dias CRS, Sá TCV, Pereira ALA, Alves CMC. Evaluation of oral condition in chronic renal patients undergoing hemodialysis. Rev Assoc Med Bras. 2007; 53: 510-4.

19. Borawski J, Wilczyñska-Borawska M, Stokowska W, Myœliwiec M. The periodontal status of pre-dialysis chronic kidney disease and maintenance dialysis patients. Nephrol Dial Transplant. 2007; 22: 457-64.

20. Thorman R, Neovius M, Hylander B. Clinical findings in oral health during progression of chronic kidney disease to end-stage renal disease in a Swedish population. Scand J Urol Nephrol. 2009; 43: 154-9.

21. Hamdy NAT. Calcium and Bone Metabolism Pre- and Post-Kidney Transplantation. Endocrinol Metab Clin N Am. 2007; 36: 923-35. 